\title{
Oral fluoxetine in the management of amblyopic patients aged between 10 and 40 years old: a randomized clinical trial
}

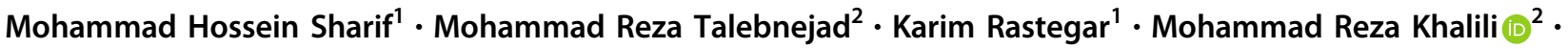 \\ M. Hossein Nowroozzadeh (iD ${ }^{2}$
}

Received: 29 April 2018 / Revised: 17 October 2018 / Accepted: 10 January 2019 / Published online: 19 February 2019

(c) The Royal College of Ophthalmologists 2019

\begin{abstract}
Objective The objective of this study is to assess the efficacy of oral fluoxetine therapy in improving the visual function of amblyopic patients aged between 10 and 40 years old.

Methods In this double-blinded, randomized, controlled trial (IRCT2016052428046N1; registered retrospectively), 40 eligible participants with anisometropic or mixed amblyopia were randomly assigned to either fluoxetine or placebo groups. Participants with anisometropia and $\log$ MAR best spectacle-corrected visual acuity (BSCVA) worse than 0.2 logMAR in the amblyopic eye or at least a two-line of difference in the BSCVA between the fellow eyes were included. Participants with significant ocular or systemic diseases were excluded. In both groups, the better eye of each patient was patched for 4-6 h a day during the study period. Participants in the treatment group were treated with oral fluoxetine for 3 months. Change in the Snellen BSCVA (after 3 months) was regarded as the primary outcome measure.

Results Data from 20 participants in the fluoxetine group and 15 participants from the placebo group were analyzed (aged 11-37 years). The magnitude of improvement in visual acuity (from baseline to 3 months after treatment) was significantly higher in the fluoxetine group $(0.240 \pm 0.068 \operatorname{logMAR} ; 2.4$ line-gain) compared with the control group $(0.120 \pm 0.086$ $\log$ MAR; 1.2 line-gain).

Conclusions This study suggests beneficial effects of fluoxetine in the management of adult and adolescent amblyopia.
\end{abstract}

\section{Introduction}

Amblyopia is the most common cause of visual impairment among children with a worldwide prevalence of about 2-3\% [1]. Various treatment strategies have been used to manage amblyopia. The most commonly used method in unilateral amblyopia is occlusion therapy. The success rate of treatment is much higher if the condition is diagnosed and treated before

Supplementary information The online version of this article (https:// doi.org/10.1038/s41433-019-0360-z) contains supplementary material, which is available to authorized users.

Mohammad Reza Talebnejad

talebnejadmr@yahoo.com

1 Department of Physiology, Shiraz University of Medical Sciences, Shiraz, Iran

2 Poostchi Ophthalmology Research Center, Department of Ophthalmology, Shiraz University of Medical Sciences, Shiraz, Iran
8-9 years of age. Several studies reported diminished clinical improvement in older subjects $[2,3]$. A "critical period" has been defined to delimit the time in which treatment of amblyopia is effective. However, this notion has been challenged by studies, which showed that amblyopia could be managed effectively in children older than 8 years of age and adolescents $[4,5]$. However, there is no doubt that the treatment becomes less effective with advancing age, due to diminished plasticity of the neural visual pathways. In principle, finding ways to enhance neural plasticity during occlusion therapy in adults may increase the effectiveness of the treatment. This strategy has been followed using levodopa with controversial results [6-11].

Fluoxetine is an Food and Drug Administrationapproved, selective serotonin receptor inhibitor (SSRI) widely used as the first-line anti-depressant in most psychiatric clinics throughout the world. The beneficial effects of SSRIs in the nerve cell growth and increased gene expression of neurotrophic factors have been shown previously [6-18]. Recent observations from animal studies suggest further advantageous effects of SSRIs in enhancing 
Fig. 1 CONSORT flow diagram of the study

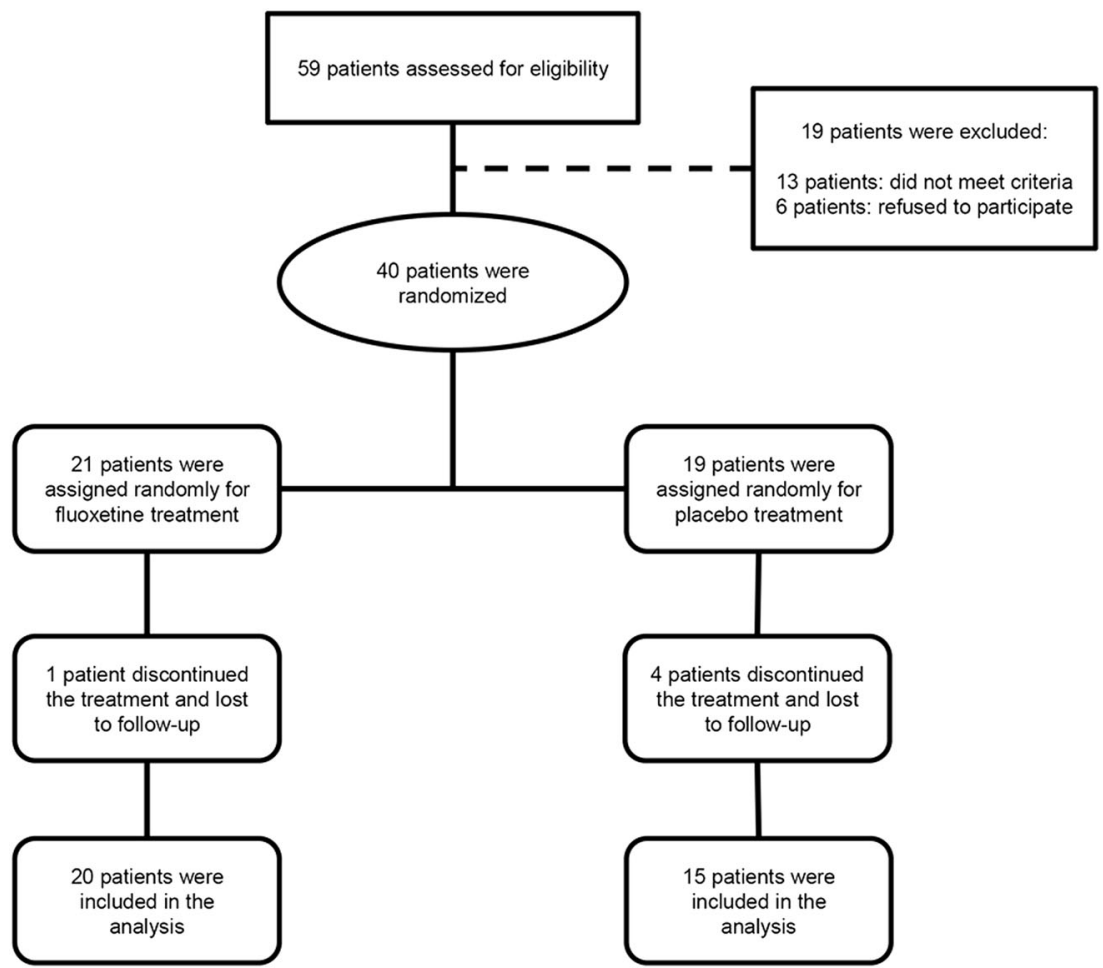

nervous system plasticity [18-22]. In addition, the beneficial effects of fluoxetine on visual functions after a critical period in an animal model have been demonstrated [19]. The aim of the present study was to assess the functional effect of fluoxetine in addition to conventional occlusion therapy in the management of amblyopic patients after a critical period.

\section{Methods}

\section{Study design and participants}

In this double-blinded, randomized, controlled trial (IRCT2016052428046N1; registered retrospectively), 40 eligible participants with anisometropic or mixed amblyopia referred to the pediatric eye clinic affiliated to Shiraz University of Medical Sciences were randomly assigned to either the fluoxetine or placebo groups (Fig. 1). The enrollment criteria of all participants were verified by two investigators (MRT and MRK). Randomization and assignment were performed using research randomizer software (version 4.0; Urbaniak and Plous, 2011). With $90 \%$ power and at an $\alpha$-level of $0.05,18$ participants were required in each group to find a difference of $0.1 \log$ MAR visual acuity (VA) between the treatment and placebo groups. Considering a $10 \%$ possibility of non-adherence, 40 participants were included.
The inclusion criteria were defined as participants aged 10-40 years old with anisometropia (Hyperopia $>1.5 \mathrm{D}$; Astigmatism > $2 \mathrm{D}$; or Myopia $>3$ D) and $\operatorname{logMAR}$ best spectacle-corrected visual acuity (BSCVA) of worse than $0.2 \log$ MAR in the amblyopic eye or at least a two-line of difference in the BSCVA between the amblyopic and the sound eye. The acceptable BSCVA for the fellow eye was at least 20/25 (0.1 $\log$ MAR). Eyes with a history or objective sign of trauma, previous intraocular surgery, uveitis, corneal opacity or any ocular disorder other than amblyopia, and anisometropia or strabismus were excluded. Participants with any systemic disorders such as diabetes, thyroid disease, rheumatologic disease, or previous consumption of fluoxetine were excluded.

All interventions were conducted according to the tenets of the Declaration of Helsinki and the study protocol was approved by the institution's ethics committee. Written informed consents were signed by each patient (or their parents). All participants were informed about common side effects of fluoxetine such as anxiety, insomnia, nervousness, tremor, anorexia, irritability, hostility, aggressiveness, impulsivity, and psychomotor restlessness. All participants or their parents and caregivers were advised of the need for close observation and communication with us in emergence of unusual changes in behavior or suicidal ideation. Advice was given to monitor participants and report such symptoms immediately to the Psychiatry Emergency Department at the institution. 


\section{Treatment arms}

The treatment arms comprised oral fluoxetine $(0.5 \mathrm{mg} / \mathrm{kg} /$ day) vs. placebo for 3 months. In both groups, the better eye of each patient was patched for 4-6h a day during the study period. The participants were advised to apply an eye patch during activities that need high visual demand such as reading, computer works, or watching TV. Participants were blinded by requesting placebo capsules similar to those that contained fluoxetine from the same pharmacological company (Abidi Pharmaceuticals, Tehran, Iran). The examiner who assessed outcomes was blinded to the type of medication (fluoxetine vs. placebo) that was consumed by the participants. Participants' adherence to the treatment was evaluated during monthly visits using a self-reported checklist based on the monthly information on how the patient adhered to the treatment (the drug, spectacle, and patching). The cases with $<70 \%$ adherence to treatment choices were planned to be excluded from the analysis.

\section{Measurements}

All eligible cases underwent a complete ocular examination including Snellen "E"-chart VA, slit-lamp examination, dilated fundoscopy, ocular motility test, and appropriate strabismus tests (including prism-cover test and modified Krimsky test). Also, new dry and cycloplegic (using topical $1 \%$ cyclopentolate drop) refractions for all participants were performed. If the new glass resulted in better BSCVA or made more comfort for the patient, the glass would be changed and 4 months would be given to adapt to the new prescription, before starting the assigned treatment [23].

Change in the Snellen BSCVA at 3 months after treatment was regarded as the primary outcome measure. Secondary outcome measures comprised changes in stereopsis, contrast sensitivity (CS), and Visual Evoked Potentials (VEPs). We included VEP test, because several studies have shown the abnormal VEP latencies in human amblyopic eyes [24, 25]. In addition, it has been reported that improvement of P100 amplitude of the pattern-VEP test parallels the VA improvement in amblyopic eyes under occlusion therapy [26].

The BSCVA was assessed using a LCD vision tester (Treviso, Italy). The Snellen E-chart (with logarithmic spacing) was used and the line that the patient could read half or more of the optotypes was recorded as the distance VA. BSCVA values were converted to logMAR VA for statistical purposes. Titmus So-001 Stereo Fly test (Stereo Optical, Chicago, IL) was used for stereopsis tests. The stereopsis was evaluated in a range of 40-800 s/arc. CS was examined using CSV1000E test (Vector Vision, Greenville, $\mathrm{OH})$ and was recorded in four spatial frequencies of 3, 6 , 12 , and 18 cycle per degrees (cpd). VEP was evaluated using Pattern-VEP method (Roland Consult, Brandenburg an der Havel, Germany). Electrodes were placed according to the international 10/20 system; the reference electrode was placed at $50 \%$ distance between the Nasion and Inion; the active electrode was placed at $10 \%$ distance anterior to the Inion; and the ground electrode was placed at $10 \%$ distance posterior to the Nasion. Visual stimuli evaluation in VEP was performed in $15^{\circ}$ and $60^{\circ}$ of the visual field with a frequency of $1 \mathrm{~Hz}, 100$ stimulus (cycle) repetitions, and $99 \%$ contrast.

For each method, initial and follow-up examinations of all participants were performed by an experienced examiner who was masked to the treatment arms. The participants were followed for 3 months and data were collected through examinations and tests for each individual in four time slots comprising baseline (day 0), 1, 3, and 3 months after starting the treatment. The CS and pattern-VEP tests were only recorded at baseline and final (3 month) examinations. To mitigate repeatability issues of the devices, each test was performed twice for every subject in each examination and the mean of the two measurements was recorded for analysis. The study was ended upon completing every post-interventional measurement for all adherent participants.

\section{Statistical analysis}

All statistical analyses were performed using IBM SPSS Statistics software version 21 (SPSS, Inc., Chicago, IL) and MedCalc version 12.2.1 (MedCalc Software, Mariakerke, Belgium). Data are presented as mean \pm SD. The normality of data was assessed by the Kolmogorov-Smirnov test. Levene's test was used to evaluate the equality of variances. Analysis of covariance was used to compare changes in each parameter between treatment and placebo group, controlled for age, sex, and the baseline value of the measured parameter. Intragroup chronological changes in outcome measures were assessed using the repeated-measures analysis of variance test. A $P$-value of $<0.05$ was considered as statistically significant.

\section{Results}

From 59 participants who were assessed for eligibility, 6 individuals did not consent to participate and 13 cases were excluded due to other ocular comorbidities (glaucoma, microcornea, coloboma, or myelinated retinal nerve fiber layer (RNFL)), pure strabismic amblyopia, and any associated systemic disorders. In total, 40 participants were entered into randomization. Twenty-one participants were randomized into the fluoxetine group and 19 into the placebo group. One patient from the case group and four from 
the control group did not continue the treatment and lost the follow-up. According to self-reported adherence checklist, all the remaining participants had excellent adherence to the treatment. Data from 20 participants in the fluoxetine group and 15 in the placebo group were recorded and analyzed (Fig. 1). The baseline characteristics of the participants were not statistically different between groups (Table 1). The mean age of the participants was $21 \pm 8$ years (median: 19.5; range: $11-37$ ) in the fluoxetine group and $21 \pm 7$ years (median: 20; range: 12-35) in the placebo group. There were 7 cases of strabismus (5 esotropia, 2 exotropia; range: 10-30 Prism Diopters (PD); 5 in the case and 2 in the control group), which had mixed type of amblyopia. The participants was from 20/25 to 20/15. The magnitude of anisometropia was $3.1 \pm 1.3 \mathrm{D}(1.6-6.5 \mathrm{D})$ in the fluoxetine and $2.8 \pm 1.5 \mathrm{D}(1.6-7.5 \mathrm{D})$ in the control group.

Regarding the $\log$ MAR BSCVA, the magnitude of improvement in VA was significantly higher in the fluoxetine group $(0.240 \pm 0.068 \log$ MAR; 2.4 line-gain $)$ compared with the control group $(0.120 \pm 0.086 \log$ MAR; 1.2 line-gain) (mean difference: $0.120 ; 95 \%$ confidence interval: $0.067-0.173 ; P<0.001$; Fig. 2; Table 2). In addition, a constant improvement in BSCVA was observed in the fluoxetine group from the first month of

Table 1 Baseline characteristics of the participants

\begin{tabular}{llll}
\hline & Group & P-value \\
\cline { 2 - 3 } & Fluoxetine & Placebo & \\
\hline Number, $n$ & 20 & 15 & \\
Gender, M / F, $n$ & $8 / 12$ & $8 / 7$ & 0.433 \\
Age, years & $21 \pm 8$ & $21 \pm 7$ & 0.895 \\
Baseline VA, logMAR & $0.490 \pm 0.148$ & $0.493 \pm 0.138$ & 0.946 \\
Anisometropia, D & $3.1 \pm 1.3$ & $2.8 \pm 1.5$ & 0.514 \\
\hline
\end{tabular}

$F$ female, $M$ male, $V A$ visual acuity, $y$ year(s)

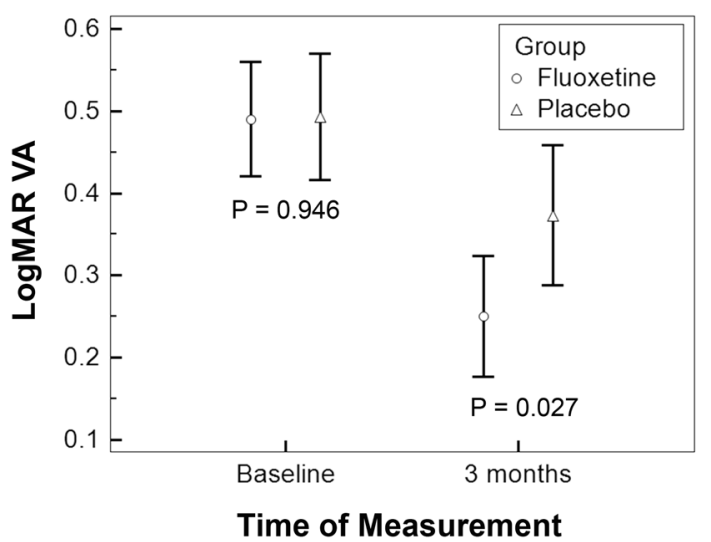
range of BSCVA of the fellow eyes from the included

treatment through the third month of treatment (Fig. 3). Stereopsis was improved from $300 \pm 278 \mathrm{~s} /$ arc at baseline to $185 \pm 188$ at 3 months in the fluoxetine group ( $P=$ $0.002)$ and from $224 \pm 94 \mathrm{~s} /$ arc to $159 \pm 89$ in the placebo group $(P=0.009)$. However, the posttreatment change in stereopsis (at 3 months) was not different between groups $(115 \pm 143 \mathrm{~s} /$ arc vs. $65 \pm 83$, respectively; $P=0.553)$. Thirty percent of participants in the treatment group and $23 \%$ of those in the placebo group gained normal stereopsis at 3 months observation.

The CS showed improvement in all frequencies after treatment in both fluoxetine and placebo groups (Supplemental Table 1). However, the magnitudes of changes in CS were not statistically different between groups in any tested frequency $(P>0.05)$, except for $3 \mathrm{cpd}$, which was more favorable in the fluoxetine group $(0.24 \pm 0.26$ vs. $0.15 \pm$ $0.24 ; P=0.004)$.

VEP measurements have also shown trends toward improvement after treatment in both groups (Supplemental Table 2). However, the magnitudes of changes in all VEP parameters were not statistically different between the two groups $(P>0.05)$.

Table 2 Comparison of visual acuity improvement after 3 months of therapy between Fluoxetine and Placebo groups

\begin{tabular}{llllll}
\hline VA improvement, log-unit & \multicolumn{2}{l}{ Group* } \\
\cline { 2 - 3 } & \multicolumn{2}{l}{ Fluoxetine } & & \multicolumn{2}{l}{ Placebo } \\
\cline { 2 - 3 } \cline { 5 - 6 } & $n$ & $\%$ & & $n$ & $\%$ \\
\hline 0 & 0 & 0 & 3 & 20.0 \\
0.1 & 2 & 10.0 & 7 & 46.6 \\
0.2 & 8 & 40.0 & 4 & 26.7 \\
0.3 & 10 & 50.0 & & 6.7 \\
Total & 20 & 100 & & 15 & 100 \\
${ }^{*} P$-value $=0.003 ; \chi^{2}$-test & & & & \\
$V A$ visual acuity & & & &
\end{tabular}

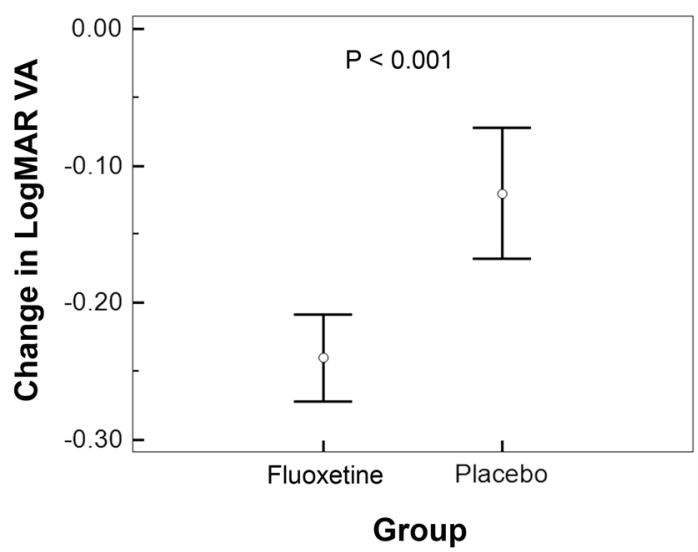

Fig. 2 Comparison of changes in the logMAR VA after treatment between the fluoxetine and placebo groups. $P$-values were calculated using independent-samples' $T$-test 


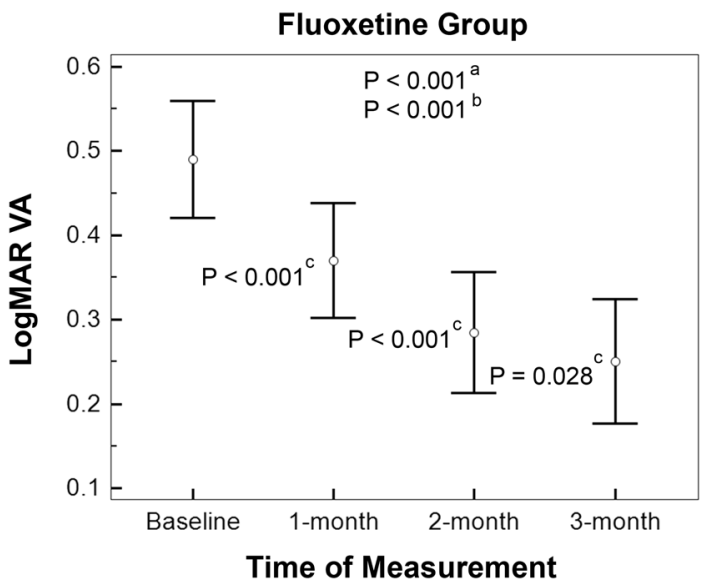

Fig. 3 Chronological changes in the logMAR VA after treatment in the fluoxetine (left) and placebo (right) groups. ${ }^{\mathrm{a}} P$-value was calculated from RMANOVA; ${ }^{b}$ the pair-wise $P$-value between the baseline and

No significant major side effect was reported by participants from using fluoxetine. Two participants receiving fluoxetine reported nausea and vomiting, and the symptoms subsided when the participants were advised to dissolve the capsule contents in juice. According to the psychiatry emergency records, there was no significant contact from any of the participants for at least 3 months after the termination of therapy.

\section{Discussion}

In this clinical trial, the beneficial effects of short-term oral fluoxetine were demonstrated in combination with the standard occlusion therapy in improving VA in amblyopia patients aged 11-37 years old compared with occlusion alone. According to the results of the present study, in terms of $\log$ MAR BSCVA, a constant improvement was observed in the fluoxetine group from the first month of treatment through the third month. Although the baseline VAs of the treatment and placebo groups were similar, the final VA at 3 months was significantly better in the fluoxetine group as compared with the placebo group. A significantly higher magnitude of improvement was also observed in the fluoxetine group; treatment group had 2.4 VA line-gain as compared with the 1.2 line-gain in the control group. The results of the present study could be explained with the aid of the findings of previous experimental studies that have shown the effects of serotonin stimulation in reinstatement of neuroplasticity [18-21]. Difficulties in the management of amblyopia beyond the critical period have been attributed to the restrictions of visual neural system plasticity to brief periods of early postnatal life. The treatment becomes less effective with advancing age, due to diminished plasticity of the neural visual pathways. Reinstatement of plasticity of

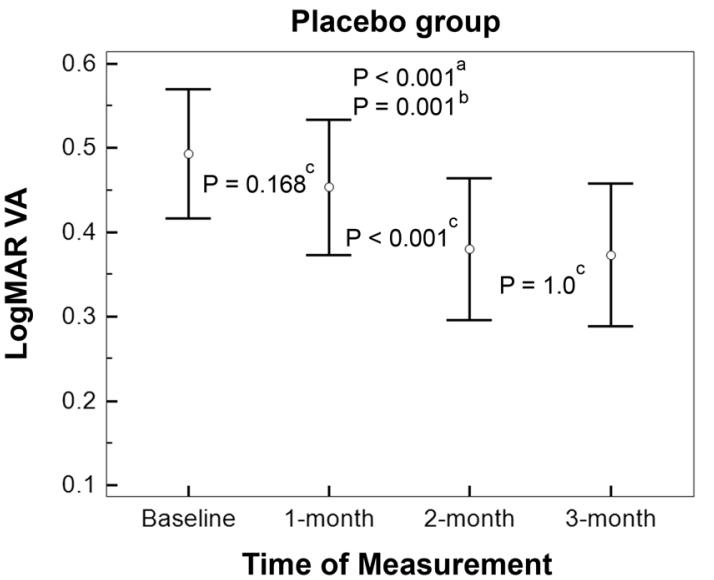

final measurements; ${ }^{c}$ the pair-wise $P$-value between the two subsequent measurements

visual pathways is the key point in management of amblyopia after the "critical period". Previous experimental studies have demonstrated the role of serotonin in reinstatement of plasticity. We think reinstatement of plasticity with fluoxetine as an SSRI and application of occlusion therapy as the conventional method of amblyopia therapy in the treatment arm of the study resulted in significant improvement in the VA in this arm.

Recent evidences from animal studies suggest the beneficial effects of SSRIs in nervous system plasticity. Serotonin regulates brain development $[22,27]$ and modulates neurotransmitter release [28]. The beneficial effects of SSRIs in nerve cell growth and increased gene expression of neurotrophic factors have been shown in previous studies [16-18]. In the experimental study performed by Baroncelli et al. [29], using both VEP and single-unit recordings, the authors showed that environmental enrichment, as a condition of increased sensory-motor stimulation, reactivates ocular dominance plasticity in visual cortex of adult rat. As they have demonstrated, serotonin is a crucial factor in this process of adult visual cortex rejuvenation, triggering a cascade of molecular events that result in the reinstatement of neural plasticity. Interestingly, it has also been shown that cortical infusion of the serotonin synthesis inhibitor neutralizes the molecular events such as reduced intracortical GABAergic inhibition and an increased brain-derived neurotrophic factor (BDNF) expression induced by environmental enrichment [29]. In another study, Vetencourt et al. [20] showed that serotonin has a key role in inducing a transitory epigenetic remodeling of chromatin structure that underlies the reactivation of plasticity in the visual system. In the mentioned study, administration of fluoxetine was found to reinstate the ocular dominance plasticity in adulthood and promote the recovery of visual functions in adult amblyopic animals. Electrophysiological studies also 
support these improvements [19]. Similar to the findings of Baroncelli et al. [29], these effects were accompanied by reduced intracortical inhibition and increased expression of the BDNF in the visual cortex $[19,29]$.

The mean VA was also observed to improve from $0.49 \pm$ 0.13 to $0.37 \pm 0.17 \operatorname{logMAR}$ after 3 months in placebo group (a statistically significant increase of $0.12 \log M A R=$ 1.2 lines of VA gain). These findings are in agreement with previous studies that demonstrated beneficial effects of patching in management of amblyopic patients after the critical period [14, 15]. Accordingly, Sen [14] found almost similar results with patching in patients after the critical period. They showed an average of 1.7 line VA improvements in 6- to 12-year-old participants and 1.2 line of VA improvement in 13- to 20-year-old subjects [14].

Therefore, this study is in line with results of previous studies that have demonstrated the effectiveness of occlusion therapy in the management of amblyopia after 7-9 years of age. In addition, it suggests better VA outcome if occlusion therapy is combined with oral fluoxetine therapy.

Our study showed that both occlusion therapy alone and combined fluoxetine/occlusion therapy increased CS in all spatial frequencies. Although the improvements were more in the fluoxetine group compared with occlusion therapy alone, this difference was only significant in $3 \mathrm{cpd}$. However, the final CS test values were still significantly lower than normal values in all the spatial frequencies in the control group; only in the lowest spatial frequencies $(3 \mathrm{cpd})$ the CS in the treatment group became statistically similar to the normal values. Previous studies [30, 31] have shown that with treatment, final CS in amblyopic patients is far from the normal. According to the present results, comparison of the two groups at 3 months showed that the only significant difference was found in the frequency of $3 \mathrm{cpd}$, which was more favorable in the fluoxetine group. This finding was speculated to be due to the fact that higher spatial frequencies are more susceptible and more frequently affected as amblyopia progresses. In addition, some investigators believed that training on CS tasks can improve VA but training on VA is not able to improve CS [12]. Huang et al [31]. confirmed that usual activities suggested for VA such as reading can serve as a stimulant for CS in lower spatial frequencies and they concluded that additional training in intermediate and high spatial frequencies may be necessary to fully recover spatial vision in amblyopia.

According to the results, stereoacuity improved in both groups in the 3 months of observations. Although the differences (post-treatment compared with pre-treatment values) were greater in the fluoxetine group than placebo, this difference was not statistically significant. It was observed that $30 \%$ of the treatment group and $23 \%$ of the placebo group gained normal stereopsis after 3 months. The observations regarding stereoacuity are in line with previous studies stating that despite early occlusion treatment only a small fraction of amblyopic eyes would gain complete stereopsis [32, 33].

As indicated by the results before treatment, P100 latencies for both $15^{\prime}$, and $60^{\prime}$ ' were significantly different from the normal values in both fluoxetine and placebo groups. This finding support previous studies that have reported increased latencies in human amblyopic eyes [24, 25]. After treatment, VEP measurements showed trends toward improvement in both groups. However, at 3-month follow-up, there was no statistically significant difference between the fluoxetine and placebo groups. After the treatment, 15' arc p100 mean latency was reduced to normal value only in the fluoxetine group and there was also a significant correlation between p100 latency reduction in $15^{\prime}$ of arc and VA improvement in the treatment group. Changes in $15^{\circ}$ of arc (smaller check size) mainly reflect the parvocellular neurosensory pathway that have smaller receptive fields and demonstrated to be affected in amblyopia [34, 35].

Although fluoxetine is approved for use in adolescent and children [36, 37], there has been a concern regarding safety profile of SSRIs; aggravation of depression and the emergence of suicidality in certain patients especially during the early phases of treatment. However, these psychological complications are more expected in the mentally ill patients rather than the healthy people who receive the medication for other purposes. Nonetheless, risk of suicidal thinking and behavior, as well as common side effects of the medication should be discussed with the patient and their parent. In addition, a close surveillance should be considered during treatment and for a while afterwards, as we did in our study. Finally, the benefit of the treatment should outweigh such complications, which need further investigations.

Study limitations include relatively small sample size, limited enrolled age group (which are not representative of the older population), using self-reported checklist for adherence to treatment (with the possibility of wrong data provided by the patient and the resultant misclassification), and short follow-up period (and also lack of follow-up after the treatment was terminated). However, this investigation could be regarded as a preliminary proof-of-concept study for using oral SSRI medications to improve the efficacy of treatment in amblyopia after the so-called critical period.

\section{Conclusion}

Based on the results of this preliminary study, the potential benefit of oral fluoxetine, a very commonly used serotonin reuptake inhibitor, to facilitate treatment outcomes in adult and adolescent amblyopia shows promise but needs further 
research. This study highlights the significance of targeting neural plasticity as an important potential arena for treatment of amblyopia in the future. This concept needs to be confirmed through future studies.

\section{Summary}

\section{What was known before}

- Amblyopia has been considered as untreatable after the critical period due to limited neural plasticity in adults.

- Minimal beneficial changes were observed after patch therapy for amblyopia in adult patients.

- Recent experimental studies showed that fluoxetine, a selective serotonin receptor inhibitor, can enhance neural plasticity.

\section{What this study adds}

- This proof-of-concept Randomised Clinical Trial (RCT) provided preliminary clinical evidence that oral fluoxetine might significantly enhance the effect of amblyopia treatment after critical period.

Acknowledgements This research funded by the Department of Physiology at Shiraz University of Medical Sciences, Grant Number GRCT00 926471.

\section{Compliance with ethical standards}

Conflict of interest The authors declare that they have no conflict of interest.

Publisher's note: Springer Nature remains neutral with regard to jurisdictional claims in published maps and institutional affiliations.

\section{References}

1. Arnold RW. Amblyopia risk factor prevalence. J Pediatr Ophthalmol Strabismus. 2013;50:213-7.

2. Gokhale AM, Gokhale SA. Effect of occlusion treatment for amblyopia at various ages. J All India Ophthalmol Soc. $1969 ; 17: 256-8$.

3. Holmes JM, Lazar EL, Melia BM, et al. Effect of age on response to amblyopia treatment in children. Arch Ophthalmol. 2011;129:1451-7.

4. Group. PEDI. A prospective, pilot study of treatment of amblyopia in children 10 to $<18$ years old. Am J Ophthalmol. 2004;137:581-3.

5. Mohan K, Saroha V, Sharma A. Successful occlusion therapy for amblyopia in 11- to 15-year-old children. J Pediatr Ophthalmol Strabismus. 2004;41:89-95.

6. Leguire LE, Rogers GL, Bremer DL, Walson PD, McGregor ML. Levodopa/carbidopa for childhood amblyopia. Invest Ophthalmol Vis Sci. 1993;34:3090-5.
7. Leguire LE, Walson PD, Rogers GL, Bremer DL, McGregor ML. Longitudinal study of levodopa/carbidopa for childhood amblyopia. J Pediatr Ophthalmol Strabismus. 1993;30:354-60.

8. Leguire LE, Komaromy KL, Nairus TM, Rogers GL. Long-term follow-up of L-dopa treatment in children with amblyopia. J Pediatr Ophthalmol Strabismus. 2002;39:326-30. quiz345-326

9. Bhartiya P, Sharma P, Biswas NR, Tandon R, Khokhar SK. Levodopa-carbidopa with occlusion in older children with amblyopia. J AAPOS. 2002;6:368-72.

10. Gore $\mathrm{C}, \mathrm{Wu} \mathrm{C}$. Medical therapies of Aamblyopia: translational research to expand our treatment armamentarium. Semin Ophthalmol. 2016;31:155-8.

11. Repka MX, Kraker RT, Dean TW, et al. A randomized trial of levodopa as treatment for residual amblyopia in older children. Ophthalmology. 2015;122:874-81.

12. Astle AT, Webb BS, McGraw PV. The pattern of learned visual improvements in adult amblyopia. Invest Ophthal Vis Sci. 2011;52:7195-204.

13. Park KH, Hwang JM, Ahn JK. Efficacy of amblyopia therapy initiated after 9 years of age. Eye (Lond). 2004;18:571-4.

14. Sen DK. Results of treatment of anisohypermetropic amblyopia without strabismus. Br J Ophthalmol. 1982;66:680-4.

15. Scheiman MM, Hertle RW, Beck RW, et al. Randomized trial of treatment of amblyopia in children aged 7 to 17 years. Arch Ophthalmol. 2005;123:437-47.

16. Manev H, Uz T, Smalheiser NR, Manev R. Antidepressants alter cell proliferation in the adult brain in vivo and in neural cultures in vitro. Eur J Pharmacol. 2001;411:67-70.

17. Malberg JE, Eisch AJ, Nestler EJ, Duman RS. Chronic antidepressant treatment increases neurogenesis in adult rat hippocampus. J Neurosci. 2000;20:9104-10.

18. Bastos EF, Marcelino JL, Amaral AR, Serfaty CA. Fluoxetineinduced plasticity in the rodent visual system. Brain Res. 1999;824:28-35.

19. Maya Vetencourt JF, Sale A, Viegi A, et al. The antidepressant fluoxetine restores plasticity in the adult visual cortex. Science. 2008;320:385-8.

20. Maya Vetencourt JF, Tiraboschi E, Spolidoro M, Castren E, Maffei L. Serotonin triggers a transient epigenetic mechanism that reinstates adult visual cortex plasticity in rats. Eur $\mathbf{J}$ Neurosci. 2011;33:49-57.

21. Gu Q, Singer W. Involvement of serotonin in developmental plasticity of kitten visual cortex. Eur J Neurosci. 1995;7:1146-53.

22. Guirado R, Sanchez-Matarredona D, Varea E, Crespo C, BlascoIbáñez JM, Nacher J. Chronic fluoxetine treatment in middle-aged rats induces changes in the expression of plasticity-related molecules and in neurogenesis. BMC Neurosci. 2012;13:5-5.

23. Stewart CE, Moseley MJ, Fielder AR, Stephens DA. Refractive adaptation in amblyopia: quantification of effect and implications for practice. Br J Ophthalmol. 2004;88:1552-6.

24. Watts PO, Neveu MM, Holder GE, Sloper JJ. Visual evoked potentials in successfully treated strabismic amblyopes and normal subjects. J AAPOS. 2002;6:389-92.

25. Talebnejad MR, Hosseinmenni S, Jafarzadehpur E, Mirzajani A, Osroosh E. Comparison of the wave amplitude of visually evoked potential in amblyopic eyes between patients with esotropia and anisometropia and a normal group. Iran J Med Sci. 2016;41:94-101.

26. Oner A, Coskun M, Evereklioglu C, Dogan H. Pattern VEP is a useful technique in monitoring the effectiveness of occlusion therapy in amblyopic eyes under occlusion therapy. Doc Ophthalmol. 2004;109:223-7.

27. Sodhi MS, Sanders-Bush E. Serotonin and brain development. Int Rev Neurobiol. 2004;59:111-74.

28. Fink KB, Gothert M. 5-HT receptor regulation of neurotransmitter release. Pharmacol Rev. 2007;59:360-417. 
29. Baroncelli L, Sale A, Viegi A, et al. Experience-dependent reactivation of ocular dominance plasticity in the adult visual cortex. Exp Neurol. 2010;226:100-9.

30. Chatzistefanou KI, Theodossiadis GP, Damanakis AG, Ladas ID, Moschos MN, Chimonidou E. Contrast sensitivity in amblyopia: the fellow eye of untreated and successfully treated amblyopes. J AAPOS. 2005;9:468-74.

31. Huang C, Tao L, Zhou Y, Lu ZL. Treated amblyopes remain deficient in spatial vision: a contrast sensitivity and external noise study. Vision Res. 2007;47:22-34.

32. Wallace DK, Lazar EL, Melia M, et al. Stereoacuity in children with anisometropic amblyopia. J AAPOS. 2011;15:455-61.

33. Stewart CE, Wallace MP, Stephens DA, Fielder AR, Moseley MJ. The effect of amblyopia treatment on stereoacuity. J AAPOS. 2013;17:166-73.
34. Shan Y, Moster ML, Roemer RA, Siegfried JB. Abnormal function of the parvocellular visual system in anisometropic amblyopia. J Pediatr Ophthalmol Strabismus. 2000;37:73-78.

35. Demirci H, Gezer A, Sezen F, Ovali T, Demiralp T, Isoglu-Alkoc U. Evaluation of the functions of the parvocellular and magnocellular pathways in strabismic amblyopia. J Pediatr Ophthalmol Strabismus. 2002;39:215-21.

36. FDA (US Food and Drug Administration). Suicidality and antidepressant drugs. Available from: https://www.fda.gov/downloa ds/drugs/drugsafety/informationbydrugclass/ucm173233.pdf.

37. Nationals Institutes of Mental Health. Antidepressant medications for children and adolescents: information for parents and caregivers. Available from: https://www.nimh.nih.gov/health/topics/child-and-a dolescent-mental-health/antidepressant-medications-for-children-a nd-adolescents-information-for-parents-and-caregivers.shtml. 